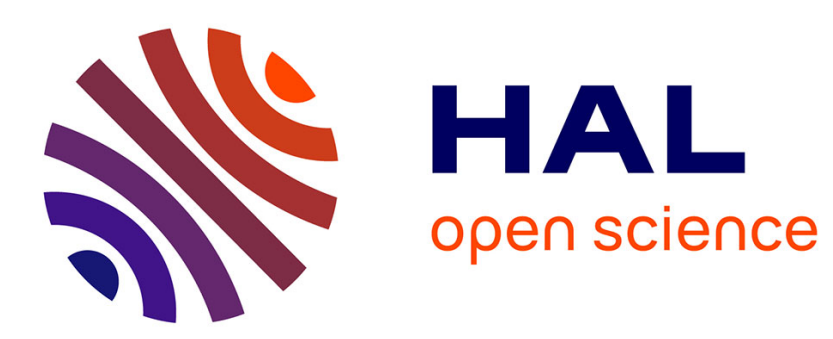

\title{
Optimized Network Slicing Proof-of-Concept with Interactive Gaming Use Case
}

Jose Jurandir Alves Esteves, Amina Boubendir, Fabice Guillemin, Pierre Sens

\section{To cite this version:}

Jose Jurandir Alves Esteves, Amina Boubendir, Fabice Guillemin, Pierre Sens. Optimized Network Slicing Proof-of-Concept with Interactive Gaming Use Case. ICIN 2020 - 23rd Conference on Innovation in Clouds, Internet and Networks and Workshops, Feb 2020, Paris, France. pp.150-152, 10.1109/ICIN48450.2020.9059328 . hal-02981083

\section{HAL Id: hal-02981083 \\ https://hal.inria.fr/hal-02981083}

Submitted on 27 Oct 2020

HAL is a multi-disciplinary open access archive for the deposit and dissemination of scientific research documents, whether they are published or not. The documents may come from teaching and research institutions in France or abroad, or from public or private research centers.
L'archive ouverte pluridisciplinaire HAL, est destinée au dépôt et à la diffusion de documents scientifiques de niveau recherche, publiés ou non, émanant des établissements d'enseignement et de recherche français ou étrangers, des laboratoires publics ou privés. 


\title{
Optimized Network Slicing Proof-of-Concept with Interactive Gaming Use Case
}

\author{
Jose Jurandir Alves Esteves* ${ }^{* \dagger}$, Amina Boubendir*, Fabice Guillemin* and Pierre Sens ${ }^{\dagger}$ \\ * Orange Labs, France \\ ${ }^{\dagger}$ Sorbonne Université / CNRS / Inria, LIP6, France \\ \{josejurandir.alvesesteves, amina.boubendir, fabrice.guillemin\}@orange.com, pierre.sens@lip6.fr
}

\begin{abstract}
Network Operators expect to accurately satisfy a wide range of user's needs by providing fully customized services relying on Network Slicing. The efficiency of Network Slicing depends on an optimized management of network resources and Quality of Service (QoS). We focus on Network Slice placement optimization problem to give an insight about a latency-aware Network Slice Placement model through a Proofof-Concept (PoC) illustrated by an Interactive Gaming timesensitive use case. Therefore, we showcase the proposed Network Slice Placement solution considering user location as a primary constraint to ensure QoS and Quality of Experience (QoE).

Index Terms-NFV, Network Slicing, Optimization, Placement Algorithms, Service Functions Chains, Gaming, Latency.

\section{Telco Network Slicing: towards Optimized DEPLOYMENTS}

Network Functions Virtualization (NFV) brings flexibility in the implementation of Network Functions (NFs) by allowing the NF logic and its hosting hardware to evolve independently [1]. With this new approach, Network Operators can deploy multiple logical networks, as a series of Virtual Network Functions (VNFs) interconnected by virtual links, over the same Physical Substrate Network composed of common hardware.

This has paved the path to the concept of Network Slicing that takes benefit of the logical and/or physical separation of network resources to allow multi-tenancy support, customization and isolation of these logical networks, called Network Slices. Optimized management and orchestration of Network Slices with their VNFs and Virtual Links (VLs) are essential to achieve the promises of Network Slicing, both for the operators (reduced costs) and the slice clients (customized services). A huge amount of work dealing with optimization problems in this context exists in the literature, especially with regard to VNF Placement and Chaining (VNFPC). This problem amounts to choosing the right servers of the Physical Substrate Network in which the VNFs composing Network Slices shall be deployed and which physical links to use in order to steer traffic between them. The problem is solved for a specific optimization objective (e.g., minimizing resource consumption) [2]-[5]. This paper describes the Proofof-Concept $(\mathrm{PoC})$ corresponding to the problem considered in a submitted full paper presenting the model [6].

We give hereafter an insight about our latency-aware approach to deal with VNF-PC using Network Slicing applied Interactive Gaming Use Case.
This use case has appeared to us as very interesting as it has recently regained popularity with the emergence of $5 \mathrm{G}$ networks and associated use cases just at the crossroad between Enhanced Mobile Broadband (eMBB) and Ultra Reliable and Low Latency Communications (uRLLC) services envisioned for this Generation. Such time-sensitive applications would benefit from Multi-Access Edge Computing (MEC) since it will allow minimal latency by locating the game server applications nearby the users [7]. Accurate latency-aware placement models are utmost important for choosing the right servers and the right links when deploying the game servers applications of a Interactive Gaming Network Slice. Hence we propose in our solution a Network Slice Placement model that takes into account the user location as an end-point of a Network Slice as a way to ensure strict latency requirements fulfilment.

The paper is organized into IV Sections. First, we provide an overview of the motivations and objectives in Section II-A. Then, we present the solution architecture in Section II-B and detail its implementation in Section II-C. The Interactive Gaming Use Case and its application to slicing is described in Section III and the planned demonstration in Section III-B. We conclude the paper in Section IV.

\section{Optimized SLiCing Proof-OF-CONCEPT}

\section{A. Overview and Description}

The objective of the proposed PoC is to show the applicability of the proposed Network Slice Placement scheme. We give some insights about the importance of taking into account user location when calculating the placement decision for the deployment of a Network Slice dedicated to Interactive Gaming applications.

We evaluate two methods: 1) the classical approach taken from the state-of-the-art, that does not take into account user location when calculating the placement of VNFs; 2) our method that, instead, considers the user location before taking a decision about the deployment. We analyse the capacity of the two models to ensure the strict latency requirements required by the Interactive Gaming Network Slice.

\section{B. Solution Architecture Description}

The architecture of our proposal is given in the Figure 1. First, we assume an Application Service Provider requests from a Network Operator a Network Slice in a B2B mode to server his own set of end users. 


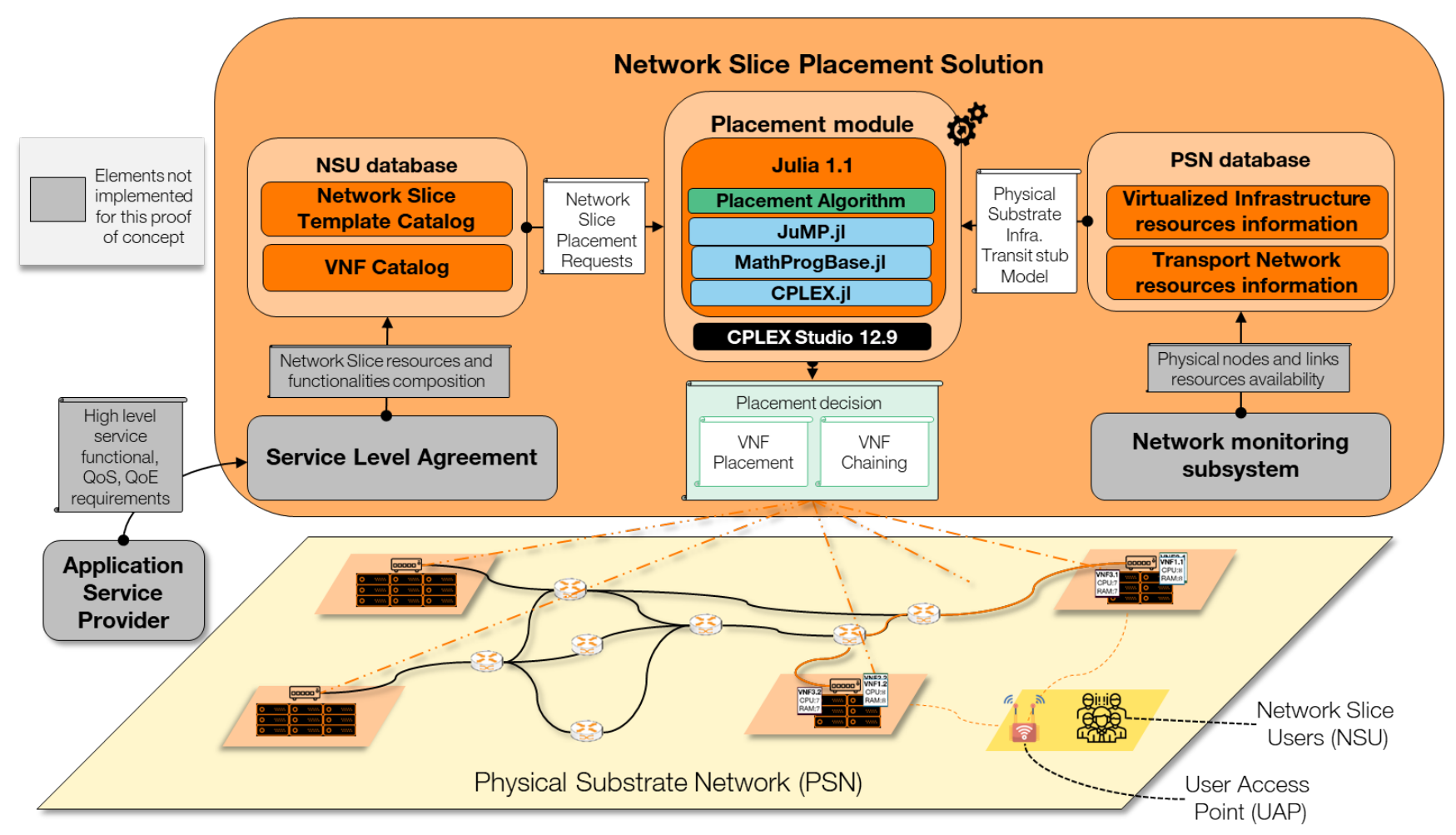

Fig. 1: Solution architecture implementation

In our use case, the Gaming Service Provider requests an Interactive Gaming Network Slice to support an Interactive Gaming Service applications to a group of gamers located in a given area like a e-Sport event. The application service provider will express some high-level (application level) QoS and $\mathrm{QoE}$ requirements that the requested network service should guarantee. These requirements are integrated to be part of the Service Level Agreement (SLA) used for the selection of VNFs and virtual links capacities which defines the Network Slice template. This template will be registered in the Network Slice Users (NSU) database. Then, the Network Slice Placement Requests and the Physical Substrate Network (PSN) Transit Stub model (containing the PSN topology and resource availability information) are used by the Placement Module so that the Placement decision is calculated using the proposed ILP-based latency-aware Placement Algorithm. This decision is to be further applied by the different Network Orchestrator layers down to the Physical Substrate Network. The PSN information is provided in real-time by the Infrastructure Monitoring System and registered into the PSN database.

\section{Solution Implementation Description \& Tools}

To allow the instantiation of our Network Slice Placement model for the Interactive Gaming Use Case, we have implemented the Placement Module, the NSU database and the PSN database. As in this PoC we consider a specific Network Slice template representing the Interactive Gaming Network Slice (see Figure 2) we do not need to implement the SLA component.
Also, as we emulate a unique execution of the placement algorithm, the monitoring system is not needed. However, for studying the Network Slice Composition, the SLA and a service composition logic need to be implemented. In a case which we have multiple placement decisions done successively in time, the Network monitoring subsystem need to be implemented to monitor the availability of network state. We used the following tools in our $\mathrm{PoC}$ implementation:

- GT-ITM: We designed a random parameter generator based on the GT-ITM [8] tool to generate the data to fulfill NSU and PSI databases. Flat Graphs are used to represent the Network Slices templates data and Transit Stub Graphs are used to represent the PSN data,

- Julia: the ILP-based Placement Algorithm used in the implementation of the Placement Module is developed using Julia [9]. The JuMP.jl and MathProgBase.jl packages are used to develop a generic ILP formulation that can be solved with any linear solver,

- CPLEX: We use the default branch-and-bound algorithm from ILOG CPLEX [10] in its 12.9 version and CPLEX.j1 package to solve our model with CPLEX.

\section{OPtimized Network SLICING fOR Interactive GAMING Use CASE}

\section{A. Interactive Gaming Use Case Description}

We consider an Interactive Gaming event in which different gamers play a multi-player game and access simultaneously a gaming service application. Figure 2 summarizes the elements of the Use Case considered for the study. 
We consider an Interactive Gaming Network Slice composed of a Gateway VNF and two redundant game server applications. We assume the amount of CPU and RAM needed by the game is too high to run efficiently in the respective User Equipment (UE), there is a need for running the UE application in the network edge Clouds. The objective is to deploy this Network Slice over the PSN composed of different distributed data centers minimizing the amount of CPU, RAM and bandwidth resources consumed. Many data centers and mainly edge cloud resources might be available from a network operator with a large footprint to ensure QoS/QoE requirements fulfilment.

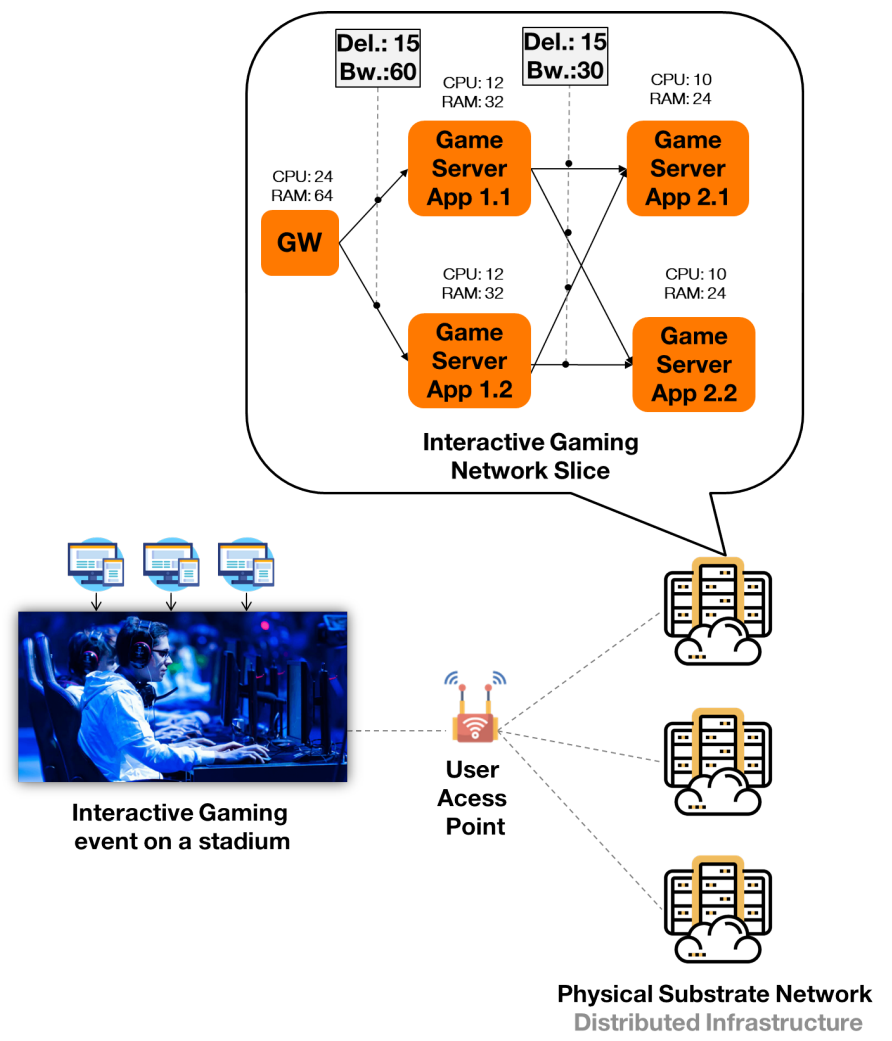

Fig. 2: Network Slicing for Interactive Gaming Use Case

\section{B. Planned Demonstration}

We will present an emulation using two scenarios of distributed PSNs: 1) a small scale scenario with 5 data centers, and 2) a medium scale scenario with 50 data centers. Each data center is composed of 5 servers. We will show the deployment of an optimal Network Slice Placement decision for the Interactive Gaming Use Case and make a comparison with an alternative method to show the visitors the advantages of our solution. We analyze the capacity of the two models in ensuring strict end-to-end latency requirements and we show a higher accuracy in the placement decision calculation. By examining the user location, we ensure the compatibility between the end-users location and the slice attachment VNFs, which is essential to achieve ultra low latency requirements. Figure 2 shows the planned demonstration setup.

\section{CONCLUSION}

We have presented in this paper a PoC of a Network Slice Placement solution proposed in a separate research paper. We mainly describe the implementation of the proposed solution architecture in order to show the feasibility and accuracy when considering the applicability of the proposed Network Slice Placement model to Interactive Gaming Use Cases.

Our approach is generic and applicable to different Network Slice use cases. Unlike the ones commonly found in the state-of-the-art, our model considers the user location when calculating the Network Slice Placement decision, allowing a guaranty of strict end-to-end latency requirements. This is utmost important in the case of highly time-sensitive applications such as e-Sports Interactive Gaming.

\section{REFERENCES}

[1] "Etsi nfv isg gs nfv 002 v1.2.1 network functions virtualization; architectural framework," 2014. [Online]. Available: https://www.etsi.org/technologies-clusters/ technologies/nfv.

[2] I. Afolabi, T. Taleb, K. Samdanis, A. Ksentini, and H. Flinck, "Network slicing and softwarization: A survey on principles, enabling technologies, and solutions," IEEE Communications Surveys \& Tutorials, vol. 20, no. 3, pp. 2429-2453, 2018.

[3] "Etsi nfv isg, etsi gs nfv-man 001 network functions virtualisation (nfv) specifications; management and orchestration," 2017.

[4] J. G. Herrera and J. F. Botero, "Resource allocation in nfv: A comprehensive survey," IEEE Transactions on Network and Service Management, vol. 13, no. 3, pp. 518-532, 2016.

[5] A. Laghrissi and T. Taleb, "A survey on the placement of virtual resources and virtual network functions," IEEE Communications Surveys \& Tutorials, vol. 21, no. 2, pp. 1409-1434, 2018.

[6] J. J. Alves Esteves, A. Boubendir, F. Guillemin, and P. Sens, "Location-based data model for optimized network slice placement," in Submitted to IEEE Network on Network Softwarization (NETSOFT 2020), 2019.

[7] "Etsi nfv isg, etsi gs mec 002 v2.1.1 multi-access edge computing (mec);phase 2: Use cases and requirements," 2018. [Online]. Available: https : / / www . etsi . org / technologies-clusters/technologies/nfv.

[8] E. W. Zegura, K. L. Calvert, and S. Bhattacharjee, "How to model an internetwork," in Proceedings of IEEE INFOCOM'96. Conference on Computer Communications, IEEE, vol. 2, 1996, pp. 594-602.

[9] J. Bezanson, S. Karpinski, V. B. Shah, and A. Edelman, "Julia: A fast dynamic language for technical computing," arXiv preprint arXiv:1209.5145, 2012.

[10] I. ILOG, “Cplex optimization studio," 2014. 